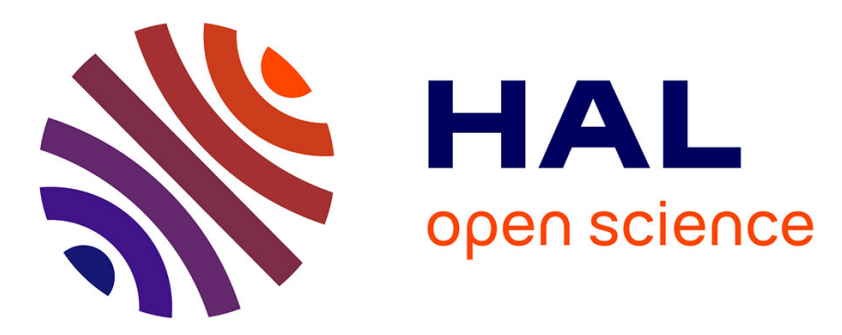

\title{
Finite-time Attractive Ellipsoid Method Using Implicit Lyapunov Functions
}

Manuel Mera, Andrey Polyakov, Wilfrid Perruquetti, Gang Zheng

\section{To cite this version:}

Manuel Mera, Andrey Polyakov, Wilfrid Perruquetti, Gang Zheng. Finite-time Attractive Ellipsoid Method Using Implicit Lyapunov Functions. 54th conference on Decision and Control, IEEE, Dec 2015, Osaka, Japan. hal-01212602

\section{HAL Id: hal-01212602 https://inria.hal.science/hal-01212602}

Submitted on 6 Oct 2015

HAL is a multi-disciplinary open access archive for the deposit and dissemination of scientific research documents, whether they are published or not. The documents may come from teaching and research institutions in France or abroad, or from public or private research centers.
L'archive ouverte pluridisciplinaire HAL, est destinée au dépôt et à la diffusion de documents scientifiques de niveau recherche, publiés ou non, émanant des établissements d'enseignement et de recherche français ou étrangers, des laboratoires publics ou privés. 


\title{
Finite-time Attractive Ellipsoid Method Using Implicit Lyapunov Functions
}

\author{
Manuel Mera*, Andrey Polyakov*,**, Wilfrid Perruquetti*,** and Gang Zheng,***
}

\begin{abstract}
A finite-time version, based on Implicit Lyapunov Functions (ILF), for the Attractive Ellipsoid Method (AEM) is developed. Based on this, a robust control scheme is presented to ensure finite-time convergence of the solutions of a chain of integrators with bounded output perturbations to a minimal ellipsoidal set. The control parameters are obtained by solving a minimization problem of the "size" of the ellipsoid subject to a set of Linear Matrix Inequalities (LMI's) constraints, and by applying the implicit function theorem. A numerical example is presented to support the implementability of these theoretical results.
\end{abstract}

\section{INTRODUCTION}

The modeling and control of dynamical systems subject to bounded perturbations is one of the most relevant problems in control theory, this is because it is unrealistic to expect actual dynamical systems to be free of noise, or that mathematical models represent perfectly, without uncertainties, real systems. For this reason the research done in the robust control area has been extensive.

The minimization of the effects due to external perturbations and uncertainties, on the performances of dynamical systems is a well-known area where several results have been obtained, and a considerable amount of different approaches have been developed, such as sliding-mode control [27], which in general only works for matched perturbations; $H_{\infty}$ Control [16], which usually asks for a vanishing condition on the perturbations; and neural networks [8], which has some implementability issues. Among these a remarkable one is the Attractive Ellipsoid Method (AEM).

The main advantages of the AEM reside in the fact that it is applicable to systems with unmatched and non-vanishing perturbations, also the control gain is usually obtained by the solution of a linear minimization problem, this is a minimization problem with a linear objective function and linear constraints, this suggests an advantage on the computational aspect of the controller design and its implementability.

The history of this approach can be track down to the late 60 's and early 70's, when the characterization of uncertain dynamics by ellipsoidal sets was first introduced in [25] and [2]. Then, the use of ellipsoids as estimations of sets guaranteed to contain a significant variable was further developed in [10], [4], [18]. The concept of the asymptotically attractive (invariant) ellipsoid as used in this paper was formalized in [26], [19] for linear systems and later extended to nonlinear systems in [23], [13] and [24].

\footnotetext{
* Non-A INRIA-LNE, Parc Scientifique de la Haute Borne 40, avenue Halley Bat.A, Park Plaza, 59650 Villeneuve d'Ascq, France

${ }^{* *}$ CRIStAL (UMR CNRS 9189), Ecole Centrale de Lille, BP 48, Cité Scientifique, 59651 Villeneuve-d' Ascq, France
}

The AEM is based on [6] the Lyapunov analysis, so it is natural to use the Implicit Lyapunov Function (ILF) method, originally presented in [9], and later revisited in [1], [20], to extend this approach and obtain additional features such as finite-time convergence to the ellipsoidal region.

This is a very desirable property considering that many applications require control algorithms fast enough to guarantee the convergence of system dynamics to a desirable value or set of values in finite-time. The finite-time stabilization [14] is crucial when the transient dynamics are required to end as fast as possible or in a specific time interval, as in robotics and aeronautics. Some examples of this can be found in [3], [7] and [15].

The ILF, as presented in [20], [21], [22], is used to construct a robust control strategy by using a Lyapunov function defined implicitly in an algebraic equation. The stability analysis then does not require the explicit solution of this equation, instead the stability conditions can be revised directly using the implicit function theorem (see [5]).

To simplify this analysis an homogeneous function is proposed. This is because homogeneity is a very useful property in the study of finite-time stability (see [28], [12], [17]). Specifically, if an asymptotically stable system is homogeneous of negative degree, then it is finite-time stable.

The AEM with the ILF derives in a minimization problem of the size (trace) of the ellipsoid characterizing matrix subject to a set of constraints obtained from the finite-time stability conditions, which can be expressed as a set of Linear Matrix Inequalities (LMI's). The implementable control strategy is obtained from the solution of this optimization problem, and the application of a numerical procedure.

\section{Problem Statement}

Considering the system

$$
\begin{aligned}
& \dot{x}(t)=A x(t)+b u(t), \\
& y(t)=x(t)+\xi(t),
\end{aligned}
$$

where $x \in \mathbb{R}^{n}$ is the state vector, $u \in \mathbb{R}$ is the control input, $y \in \mathbb{R}^{n}$ is the measurable output, $\xi \in \mathbb{R}^{n}$ is an unknown but bounded perturbation,

$$
\xi(t)^{T} Q \xi(t) \leq 1, \forall t \geq 0,
$$

with $Q=Q^{T}>0$, no additional restriction is imposed on $\xi$, however it is not considered to be random,

$$
A=\left(\begin{array}{cccc}
0 & 1 & \cdots & 0 \\
\vdots & \vdots & \ddots & \vdots \\
0 & 0 & \cdots & 1 \\
0 & 0 & \cdots & 0
\end{array}\right), \text { and } b=\left(\begin{array}{c}
0 \\
\vdots \\
0 \\
1
\end{array}\right)
$$


The objective of this paper is to design a robust feedback control strategy for the system (1) that ensures finite-time convergence of the system solutions to an ellipsoidal region despite having noisy measurements.

The ILF approach is used to develop an extended version of the AEM which includes finite-time convergence to the ellipsoid. The main tool for these two approaches is the Lyapunov function method, which in this case is implicitly defined as a solution of an algebraic equation. The analysis of the ellipsoidal set attractivity does not require the solution of this equation, because it is possible to use the implicit function theorem to check all the required conditions directly from the implicit formulation.

\section{PRELIMINARIES}

\section{A. Finite-Time Attractive Ellipsoid}

Considering the system of the form

$$
\dot{x}(t)=f(x(t), \xi(t)), \quad x(0)=x_{0}, \quad \xi(0)=\xi_{0} ;
$$

where

- $x(t) \in \mathbb{R}^{n}$ is the state vector,

- $\xi(t) \in \mathbb{R}^{n}$ is an unknown but bounded perturbation vector

$$
\|\xi(t)\| \leq l_{0}, \forall t \geq 0, l_{0} \in \mathbb{R}_{+},
$$

- $f: \mathbb{R}^{n} \rightarrow \mathbb{R}^{n}$ is a nonlinear continuous vector field.

Assume that the following ellipsoid

$$
\mathscr{E}_{x}:=\left\{x \in \mathbb{R}^{n}: x^{T} \tilde{P}^{-1} x \leq 1\right\}, \quad \tilde{P}=\tilde{P}^{T}>0 .
$$

is an invariant set of (3). Additionally we consider the notation, for any $\theta \in \mathbb{R}^{n}$

$$
\|\theta\|_{\mathscr{E}_{x}}:=d\left(\theta, \mathscr{E}_{x}\right)=\inf _{\eta \in \mathscr{E}_{x}} d(\theta, \eta),
$$

for distance from a point $\theta$ to a set $\mathscr{E}_{x}$.

Definition 1: [11] The set $\mathscr{E}_{x}$, is an asymptotically attractive set for the system (3) if $\left\|x\left(t, x_{0}\right)\right\|_{\mathscr{E}_{x}} \rightarrow 0$, as $t \rightarrow \infty$, for any $x_{0} \in \mathbb{R}^{n}$.

The asymptotic attractivity of $\mathscr{E}_{x}$ guarantees convergence of the system solutions, with any initial condition $x_{0}$, to the ellipsoid. However, it is always desirable to have some estimation for the time of convergence to the set. Addressing this, the definition of finite-time attractive ellipsoid is presented.

Definition 2: The set $\mathscr{E}_{x}$ is a finite-time attractive ellipsoid of system (3) if it is an asymptotically attractive ellipsoid, and there exists a function $T$ (called the settling time function): $\mathbb{R}^{n} \backslash \mathscr{E}_{x} \rightarrow \mathbb{R}_{+}$such that for any $x_{0} \notin \mathscr{E}_{x}$ (i.e. $x_{0} \in \mathbb{R}^{n}: x_{0}^{T} \tilde{P}^{-1} x_{0}>1$ )

$$
\lim _{t \rightarrow T\left(x_{0}\right)}\left\|x\left(t, x_{0}\right)\right\|_{\mathscr{E}_{x}}=0
$$

in terms of the matrix $\tilde{P}$ this means

$$
\varlimsup_{t \rightarrow T\left(x_{0}\right)} x^{T}\left(t, x_{0}\right) \tilde{P}^{-1} x\left(t, x_{0}\right) \leq 1 .
$$

Note that the Definitions 1 and 2 do not ask for stability of the set, only consider the attractivity property.

\section{B. Implicit Lyapunov Method for Attractive Ellipsoid}

For the very specific case when $\xi(t)=0, \forall t$, we have:

Theorem 1 ([21]): If there exists a continuous function

$$
\begin{aligned}
& G: \mathbb{R}_{+} \times \mathbb{R}^{n} \rightarrow \mathbb{R} \\
& (V, x) \rightarrow G(V, x)
\end{aligned}
$$

satisfying the conditions

C1) $G$ is continuously differentiable outside the origin for all positive $V \in \mathbb{R}_{+}$and for all $x \in \mathbb{R}^{n} \backslash\{0\}$;

C2) for any $x \in \mathbb{R}^{n} \backslash\{0\}$ there exists $V \in \mathbb{R}_{+}$such that

$$
G(V, x)=0 ;
$$

C3) let $\Phi=\left\{(V, x) \in \mathbb{R}_{+} \times \mathbb{R}^{n} \backslash\{0\}: G(V, x)=0\right\}$, then, $\forall(V, x) \in \Phi$

$$
\lim _{\|x\| \rightarrow 0} V=0^{+}, \lim _{V \rightarrow 0}\|x\|=0, \lim _{\|x\| \rightarrow \infty} V=+\infty ;
$$

C4) the inequality $\frac{\partial G(V, x)}{\partial V}<0$ holds for all $V \in \mathbb{R}_{+}$and for all $x \in \mathbb{R}^{n} \backslash\{0\}$;

C5) $\frac{\partial G(V, x)}{\partial x} f(x, 0)<0$ holds for all $(V, x) \in \Phi$;

then the origin of (3) is globally uniformly asymptotically stable.

Theorem 2 ([21]): If there exists a continuous function $G: \mathbb{R}_{+} \times \mathbb{R}^{n} \rightarrow \mathbb{R}$ that satisfies the conditions C1)-C4) of Theorem 1 and the condition

C5bis) there exist $c>0$ and $0<\mu<1$ such that $\frac{\partial G(V, x)}{\partial x} f(x, 0) \leq c V^{1-\mu} \frac{\partial G(V, x)}{\partial V}$ for $(V, x) \in \Phi$, then the origin of the system (3) is globally uniformly finite-time stable and $T\left(x_{0}\right) \leq \frac{V_{0}^{\mu}}{c \mu}$ is the settling time function, where $G\left(V_{0}, x_{0}\right)=0$.

Now considering the more general case when $\|\xi(t)\| \leq$ $l_{0} \forall t$ in (3), it is unrealistic to expect that the system trajectories converge to the origin. Still, it is possible for the system trajectories to converge to a certain set depending on $l_{0}$, the bound of the perturbations. In order to define this set in terms of the conditions given by the ILF we present the next Corollary.

Corollary 1: Let the conditions C1)-C4) from Theorem 1 hold. If the inequality $\frac{\partial G(V, x)}{\partial x} f(x, \xi)<0$ holds for all $(V, x) \in$ $\Phi$ and all $\|\xi\| \leq l_{0}$ such that $V(x)>1$, then the set

$$
\mathscr{W}:=\left\{x \in \mathbb{R}^{n}:(V, x) \in \Phi, 0<V \leq 1\right\} \cup\{0\},
$$

is a globally asymptotically stable set of (3).

These conditions repeat in an implicit form the requirements for the Lyapunov-like (storage) function of the AEM, this is a positive function which is not necessarily monotonically non-increasing in all $\mathbb{R}^{n}$, but only outside of a bounded set. Conditions $\mathrm{C} 1, \mathrm{C} 2, \mathrm{C} 4$ and the implicit function theorem [5], denote that the equation $G(V, x)=0$ defines a unique function $V: \mathbb{R}^{n} \backslash\{0\} \rightarrow \mathbb{R}_{+}$for all $x \in \mathbb{R}^{n} \backslash\{0\}$. The function $V$ is continuously differentiable outside the origin and $\frac{\partial V}{\partial x}=-\left[\frac{\partial G(V, x)}{\partial V}\right]^{-1} \frac{\partial G(V, x)}{\partial x}$ for $G(V, x)=0, \forall x \neq 0$. The implicit function theorem and conditions C3, C4 and Corollary 1 prove that $\dot{V} \leq 0$, while $V>1$. 
The next corollary specifies the finite-time attractive set.

Corollary 2: If there exists a continuous function $G$ : $\mathbb{R}_{+} \times \mathbb{R}^{n}$ that satisfies the conditions $\mathrm{C} 1-\mathrm{C} 4$, the one in Corollary 1, and additionally it fulfills that there exists $c>0$ and $\mu \in(0,1)$ such that $\frac{\partial G(V, x)}{\partial x} f(x, \xi) \leq-c V^{1-\mu} \frac{\partial G(V, x)}{\partial V}$ for $(V, x) \in \Phi$ and $V(x)>1$, then $\mathscr{W}$ is a globally finite-time attractive set for system (3), and $T\left(x_{0}\right) \leq \frac{V_{0}^{\mu}-1}{c \mu}$ is the settling time estimation with $G\left(V_{0}, x_{0}\right)=0$.

One of the main features of the control design using the AEM is the possibility to minimize in certain sense the ellipsoid size, insuring that the system solutions converge to a minimal set in $\mathbb{R}^{n}$ regardless of perturbations or uncertainties [24]. The usual characterization of the size of the ellipsoid in the AEM is conveniently described by the trace of the matrix $\tilde{P}$. The "natural" restrictions of the system and its parameters are written in the form of LMI's. Hence, the control parameters that ensure convergence to this minimal set are obtained through the solution of a linear minimization problem which objective function is $\operatorname{tr}(\tilde{P})$, and the corresponding constraints are a set of LMI's. The same approach is used in the next sections to obtain a characterization of the minimal finitetime version of the Attractive Ellipsoid.

\section{Control Design}

\section{A. Finite-time attractive set}

Let us consider the following ILF [20]

$$
G(V, x):=x^{T} D_{r}\left(V^{-1}\right) P D_{r}\left(V^{-1}\right) x-1,
$$

where $V \in \mathbb{R}_{+}, x \in \mathbb{R}^{n}, P=P^{T} \in \mathbb{R}^{n \times n}, P>0$,

$$
D_{r}(\lambda):=\operatorname{diag}\left\{\lambda^{r_{i}}\right\}
$$

with

$$
r_{i}=1+(n-i) \mu, i \in\{1, \ldots, n\}, 0<\mu<1 .
$$

Denoting

$$
H_{\mu}:=\operatorname{diag}\left\{r_{i}\right\},
$$

and defining $V_{y}:=V(y)$.

Note that when $\mu=0$ equation (5) coincides with the storage function in the AEM.

Theorem 3: Let $\mu \in(0,1)$, and some tunning parameters $\delta$ and $\beta$ such that $\delta>1, \beta \in \mathbb{R}_{+}$, the matrix

$$
\Omega=\left(\begin{array}{cccc}
A X+b Y+X A^{T}+Y^{T} b^{T} & b Y & b Y & X \tilde{D}_{m} \\
+H_{\mu} X+X H_{\mu} & -\underline{\gamma}^{-2+2 \mu} \delta X & 0 & 0 \\
Y^{T} b^{T} & 0 & -\beta X & 0 \\
Y^{T} b^{T} & 0 & 0 & -\frac{r_{1}}{\beta} I_{n} \\
\tilde{D}_{m} X & & &
\end{array}\right),
$$

where $X=X^{T} \in \mathbb{R}^{n \times n}, Y \in \mathbb{R}^{1 \times n}$ and $\tilde{D}_{m} \in \mathbb{R}^{n \times n}$ is a diagonal matrix which its $i-$ th entry $m_{i}$ is given by

$$
m_{i}:=\max \left\{\left|\underline{\gamma}^{-(n-i+1) \mu}-1\right|,\left|\bar{\gamma}^{-(n-i+1) \mu}-1\right|\right\},
$$

for $i \in\{1, \ldots, n\}$, and

$$
\underline{\gamma}=\sqrt{\frac{r_{1}}{r_{2}}}\left(1-\sqrt{\frac{r_{1}}{r_{3}}}\right), \bar{\gamma}=\sqrt{\frac{r_{2}}{r_{1}}}\left(1+\sqrt{\frac{r_{1}}{r_{3}}}\right),
$$

with $r_{1}:=1 / \lambda_{\max }(X), r_{2}:=1 / \lambda_{\min }(X)$, and $r_{3}:=\lambda_{\min }(Q)$. If the LMI system

$$
\left\{\begin{array}{l}
0<Q^{-1} \leq X \\
0<\delta^{2} X \leq X H_{\mu}+H_{\mu} X \\
0<Q H_{\mu}+H_{\mu} Q \\
\Omega \leq 0
\end{array}\right.
$$

is feasible for some $X$ and $Y$; the control input

$$
u\left(V_{y}, y\right)=V_{y}^{1-\mu} k D_{r}\left(V_{y}^{-1}\right) y,
$$

where $k:=Y X^{-1}, V_{y} \in \mathbb{R}_{+}$is such that $G\left(V_{y}, y\right)=0$, with $G$ defined as in (5).

Then the ellipsoid (4), with $\tilde{P}=D_{r}(\bar{\gamma}) X D_{r}(\bar{\gamma})$ is a finitetime attractive ellipsoid for the closed-loop system (1) with the settling time function estimation given by $T\left(x_{0}\right) \leq$ $\frac{1}{1-\delta^{-1}} \bar{\gamma}^{\mu}\left(V^{\mu}\left(y_{0}\right)-1\right)$.

\section{B. Minimization of the Attractive Ellipsoid}

Let us consider the minimization of the ellipsoidal set described in Theorem 3. In order to do so we minimize the trace of the matrix $X$ subject to the constraints defined in the LMI's system, as usual. Note that the minimization of $X$ is equivalent to the minimization of the ellipsoidal set characterized by $D_{r}\left(\bar{\gamma}^{-1}\right) P D_{r}\left(\bar{\gamma}^{-1}\right)$. Then, the minimization problem statement is the following

$$
\operatorname{tr}(X) \rightarrow \min _{X, Y}
$$

subject to (6)

\section{On the boundedness of the control}

If it is necessary to restrict the magnitude of the control signal inside the ellipsoid, it is sufficient to include an additional constraint

$$
\sigma^{-2} k^{T} k \leq P
$$

to the optimization problem (8). This, in terms of the matrices $X$ and $Y$ and using the Schur's complement can be represented in $\mathrm{LMI}$ form as

$$
\left(\begin{array}{ll}
X & Y^{T} \\
Y & \sigma^{2}
\end{array}\right) \geq 0
$$

this inequality implies that for any trajectory inside the ellipsoid $\left(V_{y} \leq 1\right)$ we will have

$$
\begin{aligned}
\|u\|^{2} & =V_{y}^{2-2 \mu} y^{T} D_{r}\left(V_{y}^{-1}\right) k^{T} k D_{r}\left(V_{y}^{-1}\right) y \\
& \leq \sigma^{2} V_{y}^{2-2 \mu} y^{T} D_{r}\left(V_{y}^{-1}\right) P D_{r}\left(V_{y}^{-1}\right) y \leq \sigma^{2} V_{y}^{2-2 \mu} \leq \sigma^{2} .
\end{aligned}
$$

\section{NUMERICAL RESUlTS}

Considering a chain of integrators as in (1) with $A \in$ $\mathbb{R}^{4 \times 4}$, with the initial conditions $x_{0}=\left(\begin{array}{llll}1,1,1.1 & 1\end{array}\right)^{T}$ the selected perturbation considered was $\xi(t)=0.5+$ $0.25 \sin (100 t)+0.25 \sin (t)\left(\begin{array}{llll}0.0002, & 0.002, & 0.02 & 0.2\end{array}\right)^{T}$, and the matrix $Q$ is defined as

$$
Q=\left(\begin{array}{cccc}
24.75 & 0 & 0 & 0 \\
0 & 24.75 & 0 & 0 \\
0 & 0 & 24.75 & 0 \\
0 & 0 & 0 & 24.75
\end{array}\right)
$$


Selecting $\mu=0.4, \delta=1.5, \sigma^{2}=125$ and $\beta=1$, the ellipsoidal matrix and the matrix gain obtained from the solution of the LMI problem stated in theorem 3, are the following

$$
\begin{aligned}
\tilde{P} & =\left(\begin{array}{cccc}
0.0445 & -0.0492 & 0.0342 & -0.0004 \\
-0.0492 & 0.0680 & -0.0658 & 0.0179 \\
0.0342 & -0.0658 & 0.1005 & -0.0834 \\
-0.0004 & 0.0179 & -0.0834 & 0.2803
\end{array}\right), \\
\hat{k} & =\left(\begin{array}{llll}
-111.6465 & -121.0438 & -52.2740 & -10.3568
\end{array}\right),
\end{aligned}
$$

and $\bar{\gamma}=14.9111$. The function $V$ is calculated by the algorithm presented in [20], which is based on the bisection numerical method, to find the zeros of (5). The simulations have been done using the explicit Euler method with a sampling period with 0.001 seconds. The obtained approximation for the settling time was $T\left(x_{0}\right) \leq 18.5037$ seconds. Figure 1 and figure 2 show the states convergence to the ellipsoidal set in the $x_{1}$ vs. $x_{2}$ and $x_{3}$ vs $x_{4}$ projections respectively. Figure 3 presents the evolution of the states in time, while figure 4 shows the control output in the same way.

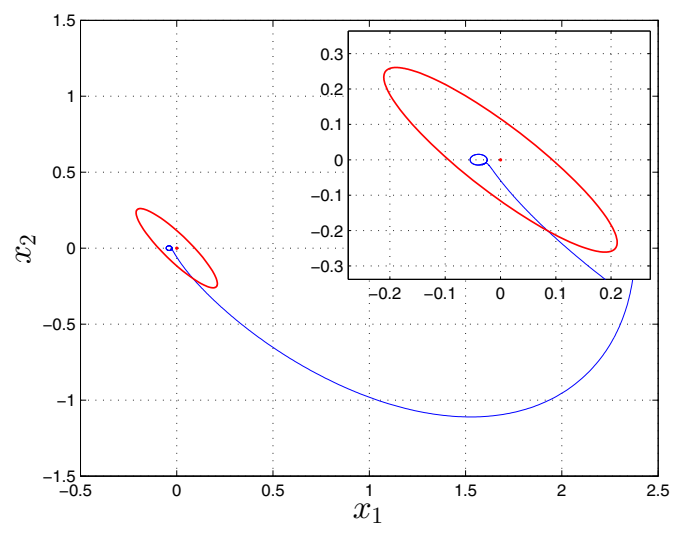

Fig. 1. System trajectories projection on the subspace $x_{1}$ vs. $x_{2}$.

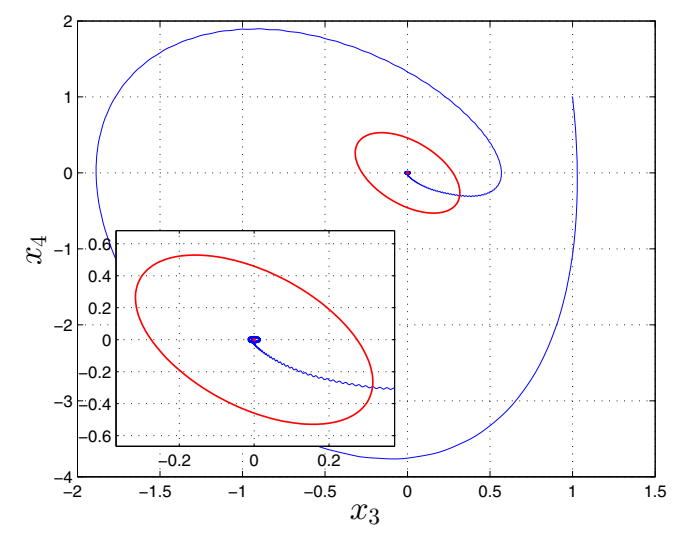

Fig. 2. System trajectories projection on the subspace $x_{3}$ vs. $x_{4}$.

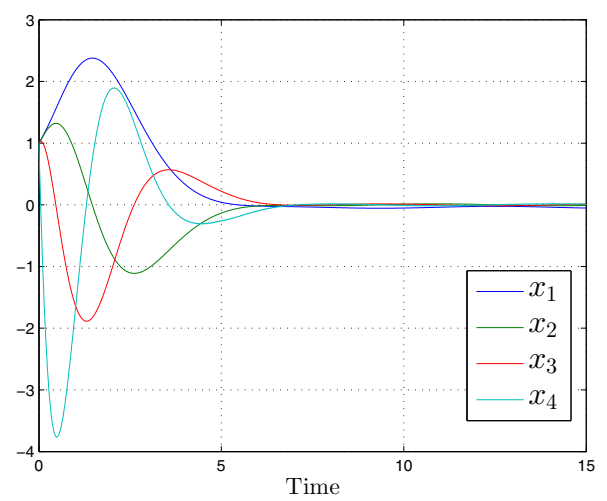

Fig. 3. States Vs. Time.

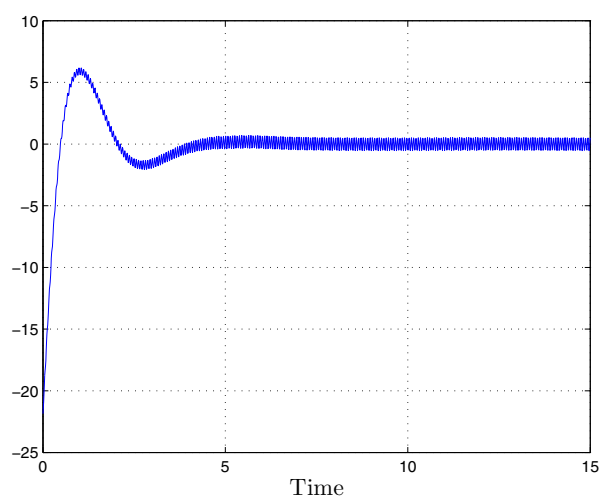

Fig. 4. Control Vs. Time.

\section{CONCLUSIONS}

A theoretical extension of the well known Attractive Ellipsoid Method was developed in this paper using the Implicit Lyapunov Function approach. Finite-time convergence despite noisy measurements to an ellipsoidal set is proved. This is achieved using a control input obtained from an ILF and the solution of a linear, respect to the matrix variables, minimization problem. The implementable numerical algorithm, based on the above extension is validated through a numerical example.

\section{REFERENCES}

[1] J. Adamy and A. Flemming. Soft variable-structure controls: a survey. Automatica, 40:1821-1844, 2004.

[2] D. Bertsekas and I. Rhodes. Recursive state estimation for a set-membership description of uncertainty. Automatic Control, IEEE Transactions on, 16(2):117-128, Apr 1971.

[3] S. Bhat and D. Bernstein. Finite time stability of continuous autonomous systems. SIAM Journal of Control and Optimization, 38(3):760-770, 1986.

[4] F. Chernousko. State estimation for dynamic systems. CRC Press, Boca Raton, 1994. 
[5] R. Courant and F. John. Introduction to Calculus and Analysis (Vol. I/II). Springer, 2000.

[6] S. Gonzalez-Garcia, A. Polyakov, and A. Poznyak. Output linear controller for a class of nonlinear systems using the invariant ellipsoid technique. In American Control Conference, 2009. ACC '09., pages 1160-1165, 2009.

[7] V. Haimo. Finite time controllers. SIAM Journal of Control and Optimization, 24(4):760-770, 1986.

[8] S. Haykin. Neural networks and learning machines. Prentice Hall, 2009.

[9] V. I. Korobov. A general approach to the solution of the bounded control synthesis problem in a controllability problem. Mat. Sb., 109(4):582606, 1979.

[10] A. B. Kurzhanskii. Control and observation under uncertainty (in Russian). Moscow, 1977.

[11] J. LaSalle. The Stability of Dynamical Systems. SIAM, 1976.

[12] A. Levant. Homogeneity approach to high-order sliding mode design. Automatica, 41(5):823-830, May 2005.

[13] M. Mera, A. Poznyak, V. Azhmyakov, and E. Fridman. Robust control for a class of continuous-time dynamical systems with sample-data outputs. In Proc. Int. Conf. on Electrical Eng., Computing Science and Automatic, pages $1-7$, Toluca, Mexico, Jan. 2009.

[14] E. Moulay and W. Perruquetti. Finite time stability and stabilization of a class of continuous systems. Journal of Mathematical Analysis and Applications, 323(2):1430 - 1443, 2006.

[15] Y. V. Orlov. Finite time stability and robust control synthesis of uncertain switched systems. SIAM Journal of Control and Optimization, 43(4):12531271, 2005.

[16] Y. V. Orlov and L. T. Aguilar. Advanced $H_{\infty}$ Control. Birkhuser, 2014.

[17] W. Perruquetti, T. Floquet, and E. Moulay. Finitetime observers: application to secure communication. IEEE Transactions on Automatic Control, 53(1):356360, 2008.

[18] B. T. Polyak, S. Nazin, C. Durieu, and E. Walter. Ellipsoidal parameter or state estimation under model uncertainty. Automatica, 40:1171 - 1179, 2004.

[19] B. T. Polyak and M. V. Topunov. Suppression of bounded exogenous disturbances: Output feedback. Automation and Remote Control, 69:801 - 818, 2008.

[20] A. Polyakov, D. Efimov, and W. Perruquetti. Finitetime stabilization using implicit Lyapunov function technique. In 9th Symposium on Nonlinear Control Systems, pages 140-145, 2013.

[21] A. Polyakov, D. Efimov, and W. Perruquetti. Homogeneous differentiator design using implicit Lyapunov function method. In Control Conference (ECC), 2014 European, pages 288-293, June 2014.

[22] A. Polyakov, D. Efimov, and W. Perruquetti. Finite-time and fixed-time stabilization: Implicit Lyapunov function approach. Automatica, 51:332340, 2015.

[23] A. Poznyak, V. Azhmyakov, and M. Mera. Practical output feedback stabilisation for a class of continuous- time dynamic systems under sample-data outputs. International Journal of Control, 84(8):1408-1416, 2011.

[24] A. Poznyak, A. Polyakov, and V. Azhmyakov. Attractive Ellipsoids in Robust Control. Springer, 2014.

[25] F. Schweppe. Recursive state estimation: Unknown but bounded errors and system inputs. Automatic Control, IEEE Transactions on, 13(1):22-28, Feb 1968.

[26] P. Usoro, F. Schweppe, D. Wormley, and L. Gould. Ellipsoidal set-theoretic control synthesis. Journal of Dynamical Systems, Measurements, and Control, 104(4):331-336, 1981.

[27] V. Utkin. Sliding Modes in Control and Optimization. Springer-Verlag, 1992.

[28] V. Zubov. Methods of A.M. Lyapunov and Their Applications. Noordhoff, 1964. 